\title{
ANALYSIS OF THE PERFORMANCE OF A LASER SCANNER FOR PREDICTIVE AUTOMOTIVE APPLICATIONS
}

\author{
J. Zeisler ${ }^{\mathrm{a}, \mathrm{b}}$, H.-G. Maas ${ }^{\mathrm{a}}$ \\ a Technische Universität Dresden, Institute of Photogrammetry and Remote Sensing, 01062 Dresden, \\ Germany - hans-gerd.maas@tu-dresden.de \\ ${ }^{\mathrm{b}}$ BMW Group, 80788 Munich, Germany - joeran.jz.zeisler@bmw.de
}

Commission III, WG III/2

KEY WORDS: LIDAR, Advanced Driver Assistance Systems, Object Detection, Bayesian Networks.

\begin{abstract}
:
In this paper we evaluate the use of a laser scanner for future advanced driver assistance systems. We focus on the important task of predicting the target vehicle for longitudinal ego vehicle control. Our motivation is to decrease the reaction time of existing systems during cut-in maneuvers of other traffic participants. A state-of-the-art laser scanner, the Ibeo Scala B2@, is presented, providing its sensing characteristics and the subsequent high level object data output. We evaluate the performance of the scanner towards object tracking with the help of a GPS real time kinematics system on a test track. Two designed scenarios show phases with constant distance and velocity as well as dynamic motion of the vehicles. We provide the results for the error in position and velocity of the scanner and furthermore, review our algorithm for target vehicle prediction. Finally we show the potential of the laser scanner with the estimated error, that leads to a decrease of up to $40 \%$ in reaction time with best conditions.
\end{abstract}

\section{INTRODUCTION}

The usage of light detection and ranging (LIDAR) in automotive application dates back to the 1990's (Watanabe et al., 1995). First implementations determined the distance and position of preceding vehicles for Adaptive Cruise Control (ACC) systems, which is still common today for Japanese manufacturers. Furthermore, there has been an extensive use of laser scanners in the field of automotive and robotic challenges, for example during the Grand (2004, 2005) and Urban Challenge (2007) of the Defense Advanced Research Projects Agency (DARPA). Detecting obstacles and identifying available free space to predict the future driving path are an example of the major tasks.

The winning team vehicle (Fig. 1c) was equipped with four different laser scanner systems with a total of 11 scanners (Darms et al., 2009). Herein, the size and number of the sensors is pointing towards the complexity of a series implementation. Besides design issues, automotive applications have to face the trade-off between performance in range, accuracy and the covered area of detection as well as cost and the specific automotive operating requirements. Cameras and radio detection and ranging systems (RADAR) were given preference up to now, particularly to gather information of relevant objects in the surrounding area of the car. Common functions are fusion-based collision mitigation and ACC systems as well as camera-only applications like sign and lane marking detection.

20 years ago, members of the Carnegie Mellon University (winners of the DAPRA Urban Challenge) initiated the first on-road field research on autonomous driving towards lateral control (Jochem et al., 1995). Looking towards future series advanced driver assistant systems (ADAS), the development of partially and highly automated functions leads to an increased demand on sensor detection performance and redundancy for long-term availability.

In this paper we give an insight to the implementation and performance of a laser scanner for a predictive task, that supports the

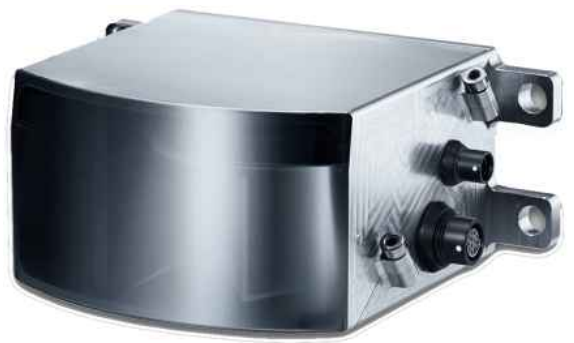

(a) Laser scanner Ibeo ScaLa B2@(Ibeo Automotive, 2015).

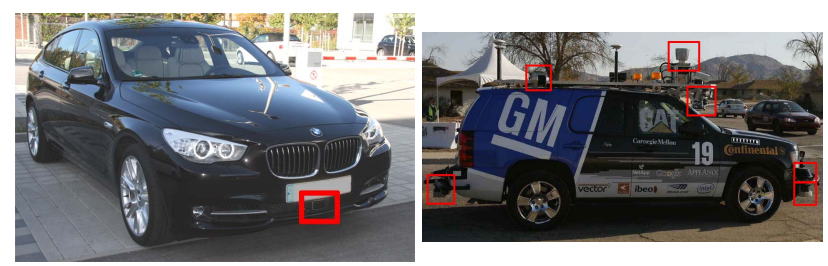

(b) Research vehicle with a front (c) Autonomous vehicle from the mounted ScaLa B2®.

DARPA Urban Challenge 2007 (Darms et al., 2009).

Figure 1: Automotive laser scanner.

longitudinal control by determining position, velocity, behavior and the resulting relevance of surrounding vehicles as target objects. First, we motivate our work, showing the current lack of detection performance of target vehicles for autonomous functions. A state-of-the-art automotive laser scanner (Fig. 1a), its sensing characteristics and the integration into a research vehicle (Fig. 1b) are presented. We validate the accuracy achieved with a test setup with the use of a GPS real time kinematics (RTK) system on a test track. Moreover, we determine an estimate for the error with the use of our current algorithm for target vehicle detection, that is reviewed consecutively. A closer insight towards the relation of sensor accuracy and the resulting detection performance is shown. Finally, we conclude with summary and outlook. 


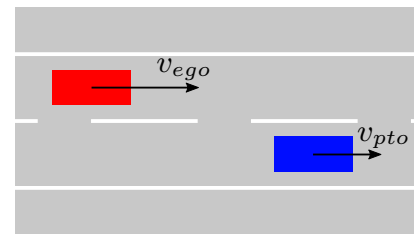

(a) Phase 0: Uniform motion of the ego vehicle (red) and potential target object PTO (blue).

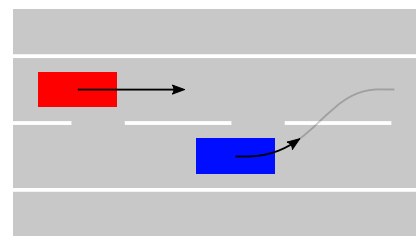

(b) Phase 1: Begin of lane change maneuver of the PTO with the projected path.

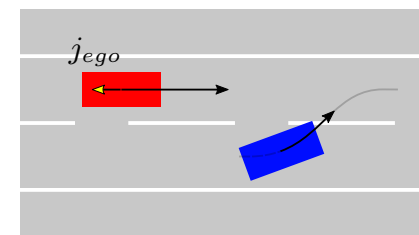

(c) Phase 2: Begin of deceleration with a defined maximum jerk (yellow) of the ego vehicle after detection of the lane change maneuver.

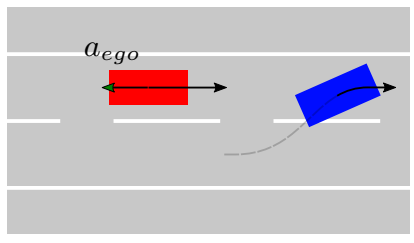

(d) Phase 3: Uniform deceleration (green) of the ego vehicle until the required minimum time gap and velocity is reached.

Figure 2: Typical lane change scenario of a potential target vehicle on a highway and the respective phases. Not drawn to scale.

\section{MOTIVATION}

The most important aspect for a safe and comfortable ADAS is to detect obstacles and traffic participants, that require an intervention in the maintained lateral or longitudinal control, as early as possible. Within our work, we focus on the task of determination and prediction of the target vehicle for longitudinal control, which has been challenging ever since the development of ACC systems, especially for close cut-in maneuvers (Winner et al., 2012). Besides avoiding collisions, the systems have to maintain a minimum time gap $\tau_{\min }$ to the preceding vehicle, presently defined in (ISO, 2009) and the respective regulations of the country. The time gap $\tau$ is determined to

$$
\tau=\frac{\Delta s}{\Delta v}
$$

where

$$
\begin{aligned}
& \Delta s: \text { longitudinal distance between the vehicles, } \\
& \Delta v \text { : velocity difference between the vehicles. }
\end{aligned}
$$

During a cut-in maneuver, the process of a lane change could be divided into four specific phases (Fig. 2), where the determination of the translational variables solely takes place in longitudinal direction:

- Phase 0: Uniform motion. The ego vehicle is traveling with a constant velocity $v_{\text {ego }}$, that is maintained by the ACC or autonomous system. There is no current target object in this example but another traffic participant in an adjacent lane, that is a potential target object (PTO). This observed vehicle is traveling at the velocity $v_{p t o_{0}}$.

- Phase 1: Begin of lane change maneuver. The ego vehicle continues with constant velocity, while the PTO starts to cut into the lane of the ego vehicle at time $t_{1}=0 \mathrm{~s}$, with the current time gap $\tau_{1}$. If at any time $t$ one has $\tau(t)<\tau_{\text {min }}$ the control needs to increase the time gap by decelerating, that is assumed in this example.

\begin{tabular}{ccc}
\hline Phase & $v_{i}(t)$ & $s_{i}(t)$ \\
\hline 1 & const. $v_{1}=v_{0}$ & $v_{1} \cdot t+s_{0}$ \\
2 & $\frac{1}{2} j \cdot t^{2}+v_{1}$ & $\frac{1}{6} j \cdot t^{3}+v_{1} \cdot t+s_{1}$ \\
3 & $a \cdot t+v_{2}$ & $\frac{1}{2} a \cdot t^{2}+v_{2} \cdot t+s_{2}$ \\
\hline
\end{tabular}

Table 1: Velocity and distance traveled of the ego vehicle at $t ; i \in 1 \ldots 3$, representing the phases. The use of index $i-1$ in the determination for phase $i$ refers to the last sample of phase $i-1$.
- Phase 2: Begin of reaction. After detection of the cut-in maneuver at $t_{2}$, the ego vehicle starts to react by decelerating, as $\tau_{2}<\tau_{\min }$. In this example and for further considerations, we assume, that the time of detection equals the moment in time, when the reaction takes place. The deceleration $a_{\text {ego }}(t)$ increases comfortably with the given jerk $j_{\text {ego }}$ (first derivative of acceleration) by: $a_{\text {ego }}(t)=j_{\text {ego }} \cdot t$ until the maximum deceleration is reached.

- Phase 3: Uniform deceleration. The ego vehicle continues decelerating after it has reached the maximum deceleration $a_{\text {ego }}$ at $t_{3}$ until $\tau \geq \tau_{\min }$.

Generally, control systems decrease the deceleration again if $\tau$ is stabilizing, which is not regarded at this point, as our main goal is to avoid a collision between the two objects under a given velocity difference $(\Delta v)$, depending on the time of reaction $\left(t_{2}\right)$. Therefore, we take a closer look at Eq. 1. Assuming the ego vehicle is traveling at a higher or equal velocity $\left(v_{\text {ego }}\left(t_{i}\right) \geq\right.$ $\left.v_{p t o}\left(t_{i}\right), i \in 0,1\right)$ and behind the PTO within the first two phases $\left(s_{\text {ego }}\left(t_{1}\right)<s_{\text {pto }}\left(t_{1}\right)\right)$, we derive the time gap $\tau(t)$ at any time by

$$
\tau(t)=\frac{\Delta s(t)}{\Delta v(t)}=\frac{s_{p t o}(t)-s_{\text {ego }}(t)}{v_{\text {ego }}(t)-v_{p t o}(t)} .
$$

We keep the velocity of the PTO constant at all time in this example: $v_{p t o}(t)=v_{p t o}$, const. and derive the traveled distance to

$$
s_{p t o}(t)=v_{p t o} \cdot t+s_{p t o_{0}},
$$

where $s_{p t o_{0}}$ is the initial distance at the start of observation.

The counterparts for the ego vehicle differ, depending on the specific phase and can be derived from Table 1 (subscript ego is omitted for better readability). Regarding a freeway scenario, we simulate the resulting minimum time gap between the ego vehicle and the PTO. Therefore, we choose a considerable set of velocity differences and we alter the detection performance within a given range for the time of detection $\left(t_{2}\right)$. Furthermore, we need an initial time gap $\left(\tau_{1}\right)$ at the time, the lane change starts and a total time for the lane change $(T L C)$ of the PTO:

$$
\begin{aligned}
v_{\text {ego } 0} & =130.0 \mathrm{~km} / \mathrm{h}, \\
a_{\text {ego }} & =-4.0 \mathrm{~m} / \mathrm{s}^{2}, \\
j_{\text {ego }} & =-2.5 \mathrm{~m} / \mathrm{s}^{3}, \\
T L C & =5.0 \mathrm{~s}, \\
\Delta v & =0.0 \ldots 50.0 \mathrm{~km} / \mathrm{h}, \\
t_{2} & =0.3 \ldots 4.0 \mathrm{~s} .
\end{aligned}
$$

The given motion parameters (velocity, deceleration and jerk) for the ego vehicle are derived from the current maximum traveling speed of a highly automated driving function (Aeberhard et al., 


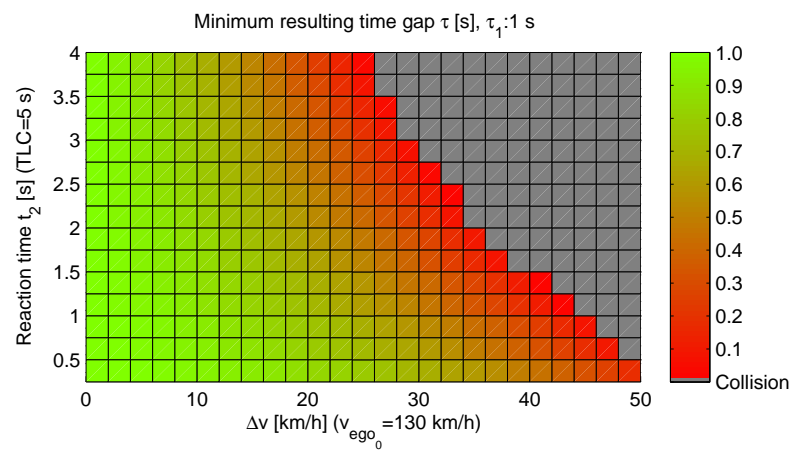

Figure 3: Simulation of the resulting minimum time gap $\tau[s]$ under given conditions. The gray areas hint towards unavoidable collisions.

2015) and research towards control laws for vehicle platoons ( $\mathrm{Li}$ et al., 1997), the average time for lane change has been examined by (Salvucci and Liu, 2002). One can see from Fig. 3, that a collision with a difference velocity of $\Delta v=50 \mathrm{~km} / \mathrm{h}$ can only be avoided, if the reaction to the maneuver takes place immediately after the start of the maneuver, which is due to the fact, that the distance of the small time gap $\tau_{1}$ is reduced very quickly. Stateof-the-art ACC systems detect objects within $t_{2} \geq 2.5 \mathrm{~s}$ under ideal circumstances (Winner et al., 2012), as the implemented logic accepts a vehicle as target object, if more than the half of the shape of the object is in the future driving path (or lane) of the ego vehicle. Difference velocities $\Delta v>30 \mathrm{~km} / \mathrm{h}$ between the ego vehicle and the PTO will lead to a collision in this case.

Within the currently given ranges of the motion parameters for the ego vehicle, it is our motivation to decrease the time until a detection of a lane change maneuver takes place and allow to handle higher velocity differences as a consequence.

\section{LASER SCANNER PERFORMANCE}

Laser scanners provide 3D information on the observed scene and allow to determine the positions of objects directly. Thus, we evaluate the performance of a laser scanner for automotive application in combination with the algorithm, that will be introduced in chapter 4. At first, we have a look at the sensor characteristics and raw data processing, followed by the accuracy evaluation of the features, that are extracted for the use in the algorithm.

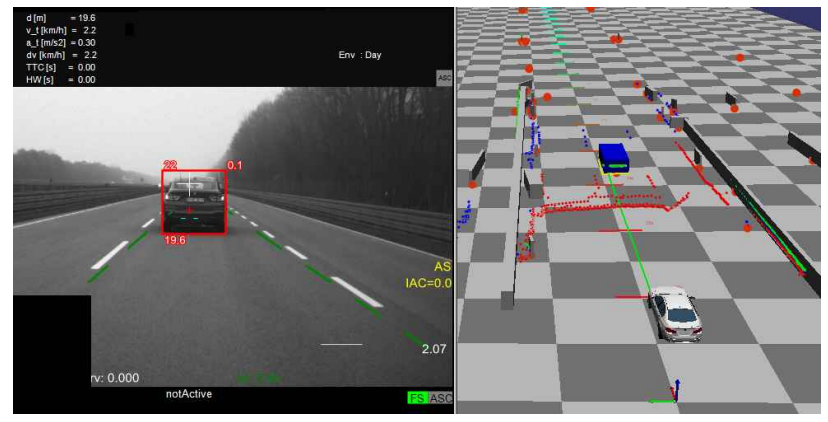

Figure 4: Camera capture (left) of a scene and visualization of scanner data (right). Ego vehicle is drawn as gray model, the classified (car, blue) target ahead. Current scan points are drawn as red, blue and green pixel. Objects reference points are red spheres. The green vector between ego and target is the reference (GPS-RTK) resultant.

\subsection{Overview of the Ibeo Scala B2 $₫$}

The Ibeo ScaLa B2@(Fig. 1a, further refered to as ScaLa) is a laser scanner, that has been developed to match the requirements of the automotive industry. As a consequent advancement of the Ibeo Lux (Velupillai and Guvenc, 2009) towards an implementation in series, it is designed as a 2D-scanner with 4 scanning layers. An in-house rotating mirror deflects the emitted beam and receives the returned echoes, which allows for a integration in the body of the vehicle (Fig. 1b). Besides raw data (3D coordinates and reflectance for every point), the sensor delivers high level data of the surrounding objects (Fig. 4). The most relevant specifications are shown in Table 2 (Ibeo Automotive, 2015).

\begin{tabular}{|c|c|c|}
\hline Feature & \multicolumn{2}{|c|}{ ScaLa } \\
\hline Scanning & horizontal & vertical \\
\hline Field of view & $145.0^{\circ}$ & $3.2^{\circ}$ (4 layers) \\
\hline Resolution/Divergence & $0.25^{\circ} / 0.10^{\circ}$ & $0.80^{\circ} / 0.80^{\circ}$ \\
\hline $\begin{array}{l}\text { Scanning } \\
\text { frequency }\end{array}$ & \multicolumn{2}{|c|}{$\begin{array}{c}25.0 \mathrm{~Hz} \text { (top and bottom layer are } \\
\text { scanned alternating at } 12.5 \mathrm{~Hz} \text {.) }\end{array}$} \\
\hline Duration of a scan & \multicolumn{2}{|c|}{$16 m s(9 m s$ flyback $)$} \\
\hline Max. output per scan & \multicolumn{2}{|c|}{1743 points } \\
\hline Distance range & \multicolumn{2}{|c|}{$0.3 \ldots<350 m$} \\
\hline Distance resolution & \multicolumn{2}{|c|}{$4.0 \mathrm{~cm}$} \\
\hline Distance error & \multicolumn{2}{|c|}{$\sigma_{d}=10.0 \mathrm{~cm}$} \\
\hline \multicolumn{3}{|l|}{ High level data } \\
\hline Reference & \multicolumn{2}{|c|}{$\begin{array}{l}\text { NTP time stamp, referring } \\
\text { to the middle of the scan. }\end{array}$} \\
\hline Object identification & \multicolumn{2}{|c|}{$\begin{array}{l}\text { Unique ID and classification (e.g. } \\
\text { Pedestrian, Bike, Car). }\end{array}$} \\
\hline Dimension & \multicolumn{2}{|c|}{$\begin{array}{l}\text { Length }(l) \text { and width }(w) \\
\text { of the bounding box. }\end{array}$} \\
\hline Position & \multicolumn{2}{|c|}{$\begin{array}{l}\text { Longitudinal and lateral } \\
\text { position }(x, y) \text { and standard } \\
\text { deviation }\left(\sigma_{x}, \sigma_{y}\right) \text { for the } \\
\text { reference point }(R P) \text {. }\end{array}$} \\
\hline Orientation & \multicolumn{2}{|c|}{$\begin{array}{l}\text { Object orientation }(\psi) \\
\text { and standard deviation }\left(\sigma_{\psi}\right) \text {. }\end{array}$} \\
\hline Velocity & \multicolumn{2}{|c|}{$\begin{array}{l}\text { Longitudinal and lateral } \\
\text { velocity }(v x, v y) \text { and standard } \\
\text { deviation }\left(\sigma_{v x}, \sigma_{v y}\right) \text {. }\end{array}$} \\
\hline
\end{tabular}

Table 2: ScaLa characteristics.

Designed as a black box system, all subsequent steps after scanning, such as the segmentation of points to objects, as well as the tracking, filtering and classification are within the know how of Ibeo. At the end of every scan, the high level object data is available in real-time. All processing takes place inside the scanner in the electronic control unit (ECU). Input of motion data (velocity and yaw rate) of the ego vehicle, and the bore-sight-alignment parameters (3D translation and rotation related to the origin of the vehicle coordinate system) is required to derive tracked object data related to the vehicle coordinate system.

Using an available CAD model of the ego vehicle (Fig. 1b), we determine the extrinsic position of the scanner during mounting in the front bumper. The yaw angle of the scanner has been determined by the use of a thin pole. Placed in sufficient distance within the longitudinal axis of the car, a single echo is returned, that should refer to the beam in the middle of the horizontal FOV. The pitch angle is calibrated by the known height of the scanner over ground and the returns from the horizontal plane, the vehicle is standing on. Determining the roll angle is neglected, since 


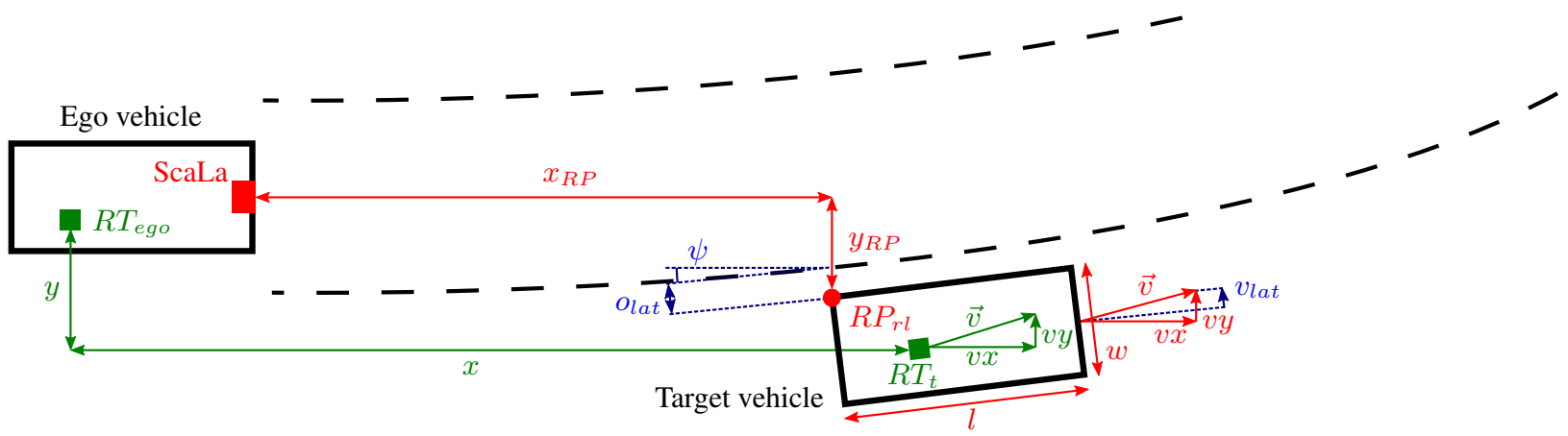

Figure 5: Test setup for performance evaluation. Green variables refer to the reference system and red to the scanner. The blue variables show the desired observations for our algorithm. Not drawn to scale.

the error during mounting is very low and has no effect on the performance. No information is given on intrinsic calibration or the accuracy of the angular deflection. Therefore, we assume the error of the given yaw angle $\alpha$, resulting from our calibration - as difference between 2 adjacent rays: $\sigma_{\alpha}=0.25^{\circ}$.

The derived high level data of the observed objects relate to the coordinate system, defined by: the $\mathrm{x}$-axis points in the driving direction of the ego vehicle and the y-axis to the left. Angular measurements are mathematically positive, starting with the $x$ axis in counter-clockwise direction towards the y-axis.

\subsection{Performance Evaluation}

In this subsection we will assess the scanner regarding the achievable performance for the approach, introduced in chapter 4.1 .

3.2.1 Error Propagation To evaluate the performance of the scanner, we need to derive information of the error of the geometric features lateral offset $\left(o_{\text {lat }}\right)$ and lateral velocity $\left(v_{\text {lat }}\right)$. Since both are not part of the scanner output, they are determined from the available object data as described in chapter 4.1. Fig. 5 shows the data derived from the scanner (red) and the required data, to determine the relevance of an object (blue). In this evaluation, we simplify the scenario, assuming a non-curved flat part of road, where the ego lane is described as a straight path $(\psi \approx 0)$. The distance $d$ to the object rear center is derived from the lateral position $y$ and the half width of the ego path $\left(w_{\text {egolane }}\right)$. As no rotations are applied to the derived object velocity vector $\vec{v}$, the lateral velocity equals the one of the object $v y$. Thus, the geometrical features are determined by

$$
\begin{aligned}
& o_{\text {lat }}=y-0.5 \cdot w_{\text {egolane }}-0.5 \cdot w, \\
& v_{\text {lat }}=v y
\end{aligned}
$$

Given Eq. 4, the standard deviations of the features could be determined by

$$
\begin{aligned}
\sigma_{o_{l a t}} & =\sqrt{\left(\sigma_{y}\right)^{2}+\left(0.5 \cdot \sigma_{w}\right)^{2}}, \\
\sigma_{v_{l a t}} & =\sigma_{v y} .
\end{aligned}
$$

3.2.2 Practical Evaluation To get an overall impression of the accuracy of the scanner under real world conditions, we set up two scenarios on a test track using a GPS-RTK system operating as reference. The ego vehicle, equipped with the scanner, follows the target vehicle, and observations from the ego vehicle scanner and the reference system are compared. Specifically, two scenarios (Fig. 6) were tested with cloudy and dry conditions:

- Scenario: Follow.

The ego vehicle follows the target vehicle in the same lane for a whole loop of the test track (6:00 min) and varies the distance within $20 \ldots 100 \mathrm{~m}$ by accelerating and decelerating. The target vehicle keeps its velocity constant at $80 \mathrm{~km} / \mathrm{h}$.

- Scenario: Sinus.

Similar to Follow, the ego vehicle stays behind the target at $80 \mathrm{~km} / \mathrm{h}$, this time keeping a constant distance of approximately $50 \mathrm{~m}$. The target vehicle performs cut-in and out maneuvers to and from the ego lane for 1:00 min on a straight part of the road.

We use a OXTS RT3000 v2 GPS-RTK system, referenced as RT, that receives input from two reference stations, located at the east and west part of the test track, which has a width of approximately $3600 \mathrm{~m}$ and a $10^{\circ}$ degree offset to the east-west axis. The a-priori standard deviations of each RT of the reference system within best conditions are (OXTS Ltd., 2015):

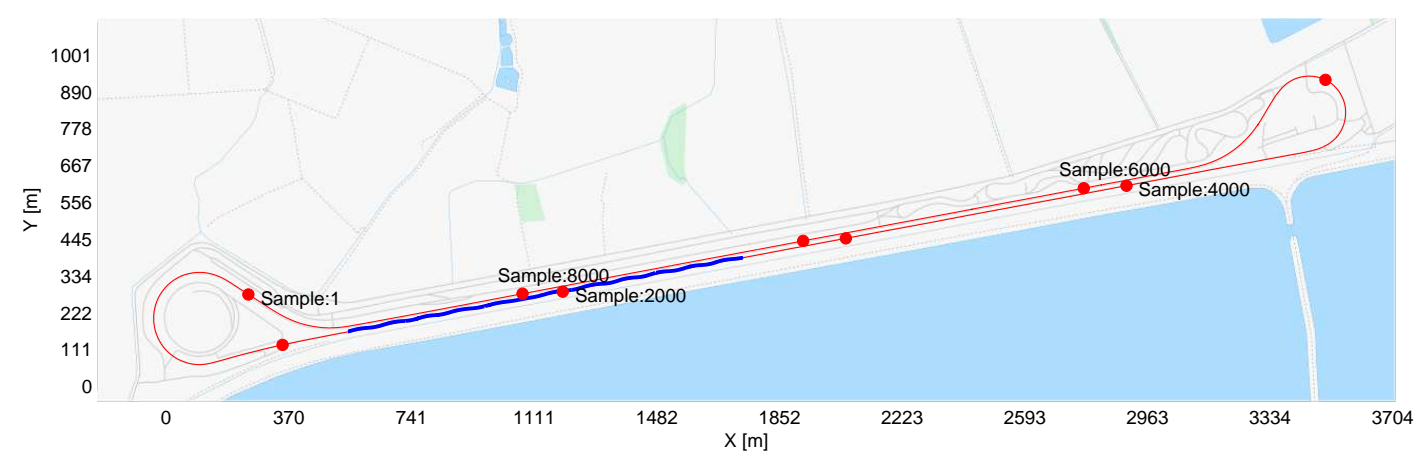

Figure 6: Overview of the scenarios (Follow: red, Sinus: blue) on the test track. A red dot is drawn for each 1000. sample of Follow. 
- $\sigma_{P o s}=1.0 \mathrm{~cm}$ for the position,

- $\sigma_{V e l}=1.4 \mathrm{~cm} / \mathrm{s}$ for the velocity,

- $\sigma_{Y a w}=0.1^{\circ}$ for the heading.

Table 3 shows the observed values, that are provided by the reference system. If we compare the resulting standard deviation for the lateral components to the one of the a-priori values, a large difference is visible, resulting from the influence of the heading, that is taken into account to determine the lateral offset and velocity as seen from the ego vehicle.

\begin{tabular}{ccc}
\hline Scenario & $\sigma_{y}^{r}$ & $\sigma_{v y}^{r}$ \\
\hline Follow & $10.0 \mathrm{~cm}$ & $4.0 \mathrm{~cm} / \mathrm{s}$ \\
Sinus & $11.0 \mathrm{~cm}$ & $4.0 \mathrm{~cm} / \mathrm{s}$ \\
\hline
\end{tabular}

Table 3: Reference system standard deviation for each scenario.

The RT systems are mounted on a fixed position in the back of each car and the bore-sight-alignement has been calibrated. As shown in Fig. 5, the position offset between the RT system for the ego vehicle $\left(R T_{\text {ego }}\right)$ and the target vehicle $\left(R T_{t}\right)$, is reduced to the position offset, as seen from the scanner. For every object the handed position refers to the reference point $R P_{h}$, where $h$ denotes eight possible positions - the four edges of the rear/front and left/right side as well as the four points in the middle of each side (E.g. $R P_{r l}$ points to the rear left RP). Time-reference is given, as the $R T$ provides the UTC stamp via the NTP interface.

To derive an estimation for the desired standard deviations $\sigma_{q}$ $\left(\sigma_{y}, \sigma_{w}\right.$ and $\left.\sigma_{v y}\right)$, we regard the uncertainties $u_{q}(k)$, at each consecutive sample $k$ between the reference $(r)$ and the scanner $(s)$ for all observations $q$ as

$$
u_{q}(k)=q^{s}(k)-q^{r}(k) .
$$

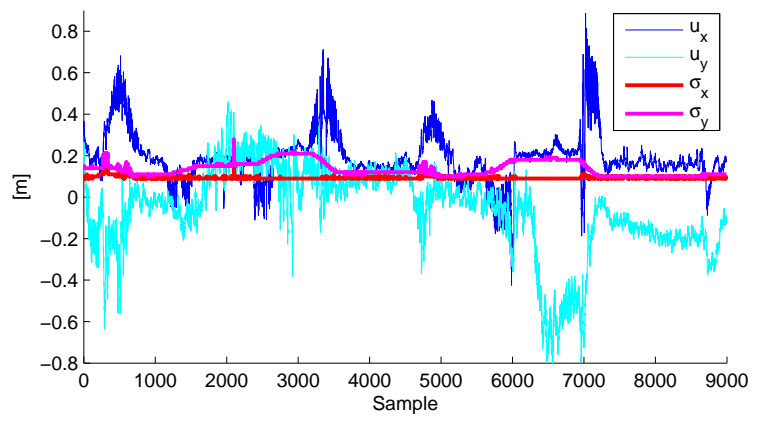

(a) Position $(x, y)$ for scenario Follow.

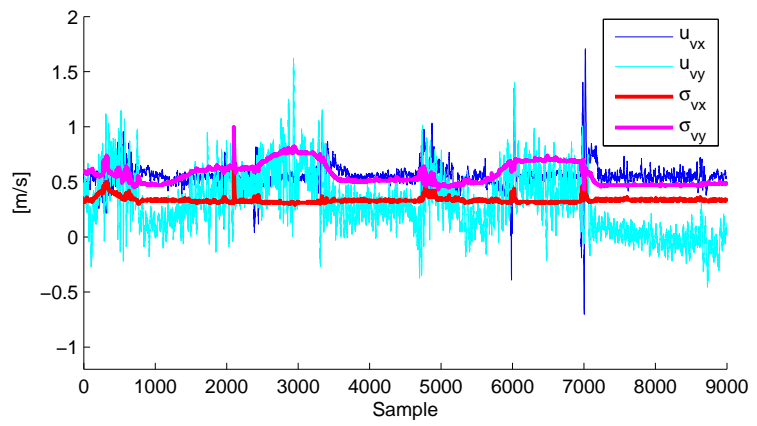

(c) Velocity $(v x, v y)$ for scenario Follow.
The derived uncertainties of the samples for distance and velocity error are shown in Fig. 7. Although the y-component is of main interest for the algorithm, we show the longitudinal parts also, as they might be of interest for further research. We provide the standard deviation estimates $\left(\sigma_{x}, \sigma_{y}, \sigma_{v x}, \sigma_{v y}\right)$, derived from object tracking of the scanner, as well. For future noise assessment (chapter 4.2), we want to evaluate if an equality between filterderived standard deviations and those, determined by reference, is given.

During both scenarios the track of the target was stable and the classification (car) valid for each sample. At a first glance an overall positive bias is visible in both scenarios for the position and velocity derived by the scanner, regarding the determined uncertainties. The estimated standard deviation of the scanner is always shown as positive value, since it results from the root of the variance. As the uncertainties to the reference show large amplitude and high frequent changes, the estimated standard deviations show a more stable behavior. Hence, the mean estimated standard deviation approximates the mean uncertainty determined.

Having a closer look at scenario Follow, the position offset shows a range between $-0.5 \ldots 0.5 \mathrm{~m}$ with peaks for longitudinal offset around samples 500, 3350, 4900 and 7000. At these samples the ego vehicle shows a deceleration and increases the distance to the target. Situations of acceleration of the ego vehicle are around sample 1300 or 2500 . The resulting negative peaks show a smaller amplitude than these for deceleration. The estimated standard deviation from tracking $\left(\sigma_{x}\right)$ doesn't represent this issue. The lateral uncertainties are around sample 500 and 6500 , where the first peak might result from the driven bend, whereas the second bend shows no influence on the lateral uncertainty. The second peak around sample 6500 is with a straight part of the road, where no deceleration applies as well, but the target is in a distance of approximately $80 \mathrm{~m}$ to the ego object. Interestingly the maximum distance of $100 \mathrm{~m}$ around sample 2900 shows

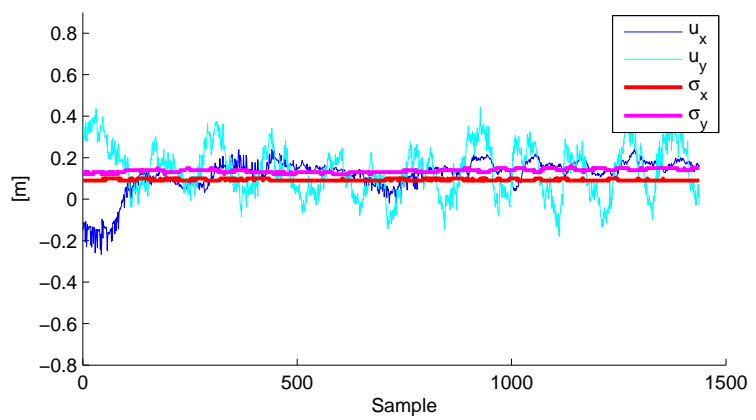

(b) Position $(x, y)$ for scenario Sinus.

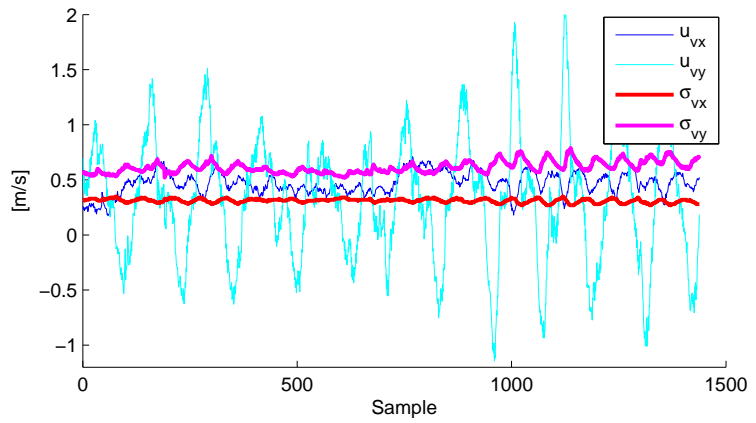

(d) Velocity $(v x, v y)$ for scenario Sinus.

Figure 7: Uncertainties $\left(u_{q}\right)$ to reference and estimated standard deviations $\left(\sigma_{q}\right)$ of the scanner for position and velocity during the scenarios. 
less influence on the lateral position uncertainty. Regarding the lateral standard deviation from tracking $\left(\sigma_{y}\right)$ the peaks aren't represented in the estimates. The peaks of the velocity uncertainties follow these of the position, where the estimated standard deviation of the scanner shows a lower value for the longitudinal part $\left(\sigma_{v x}\right)$, though representing certain peaks. A good proximity for the lateral part $\left(\sigma_{v y}\right)$ is handed.

The offset range in position, seen with Sinus is slightly smaller, but shows positive biases for $x$ and $y$ as well. One can find an alternating pattern for the lateral position offset, following the frequency of the lane changes within a range of $-0.2 \ldots 0.4 \mathrm{~m}$. Similar to Follow, the estimated standard deviations $\left(\sigma_{x}, \sigma_{y}\right)$ don't represent the derived uncertainty $\left(u_{x}, u_{y}\right)$ behavior. The lateral velocity offset seems to follow the maneuver driven as it increases and decreases with every lane change between $+2 \ldots-1 \mathrm{~m} / \mathrm{s}$. Taking a closer look at the lateral velocity, the samples show, that the maximum is reached approximately $280 \mathrm{~ms}$ later in the data of the scanner. Furthermore, the difference in the amplitude maximum shows much higher values for positive lateral velocities in the data of the scanner $\left(\max \left(v y^{s}(k+280 m s)-v y^{r}(k)\right) \approx\right.$ $1.3 \mathrm{~m})$, where as for negative lateral velocities the difference is less than the half. Comparing the estimated standard deviation of the scanner, the difference doesn't become obvious. One might take from the first evaluation, that the estimated standard deviations of the scanner should not be used for further accuracy evaluation, especially in situations with acceleration for longitudinal and lateral direction.

An estimate for the standard deviation is given by the overall uncertainty $\bar{u}_{q}\left(\sigma_{q} \approx \bar{u}_{q}\right)$, derived as RMS of the uncertainties $u_{q}(k)$ (Eq. 6) over all samples $K$ of

$$
\bar{u}_{q}=\sqrt{\frac{\sum_{k=1}^{K}\left(u_{q}(k)\right)^{2}}{K}} .
$$

We derive the uncertainties of interest, shown in Table 4. The width of the target object, determined by the scanner, was compared against the ground truth. An overall error of $10 \mathrm{~cm}$ is derived, as the scanner estimated the object slightly thinner for Follow and Sinus. Looking at the results, one can find a mostly equal uncertainty for $\bar{u}_{o_{l a t}} \approx 0.2 \mathrm{~m}$ in both scenarios. Regarding the lateral velocity $\left(v_{\text {lat }}\right)$, Sinus shows a significantly higher error, that is of interest, as typical cut-in maneuvers are similar to the ones, driven within this scenario.

\begin{tabular}{ccccc}
\hline Scenario & $\bar{u}_{y}$ & $\bar{u}_{w}$ & $\bar{u}_{\text {olat }}$ & $\bar{u}_{v y} / \bar{u}_{v_{\text {lat }}}$ \\
\hline Follow & $0.22 \mathrm{~m}$ & $0.10 \mathrm{~m}$ & $0.23 \mathrm{~m}$ & $0.40 \mathrm{~m} / \mathrm{s}$ \\
Sinus & $0.18 \mathrm{~m}$ & $0.11 \mathrm{~m}$ & $0.20 \mathrm{~m}$ & $0.66 \mathrm{~m} / \mathrm{s}$ \\
\hline
\end{tabular}

Table 4: Derived uncertainties for the scenarios.

\section{REVIEW AND ANALYSIS OF AN ALGORITHM FOR TARGET VEHICLE PREDICTION}

With the given objective (reducing the detection time) we focus on an algorithm to determine the relevance of surrounding traffic participants, such as motorcycles, cars and trucks for further classification as target objects. Consecutively, we review the functionality of a proposed algorithm, that implements a Bayesian network (BN) to determine the probability (relevance) of an observed object, and analyze the handling of sensor noise and the resulting performance, that can be achieved.

\begin{tabular}{lcccc}
\hline Lateral Evidence & \multicolumn{2}{c}{ True } & \multicolumn{2}{c}{ False } \\
Trajectory & True & False & True & False \\
\hline LBC $=$ True & 1 & 1 & 1 & 0 \\
LBC $=$ False & 0 & 0 & 0 & 1 \\
\hline
\end{tabular}

Table 5: Conditional probability table for the node Lane Boundary Crossing

\subsection{A Target Object Prediction using Bayesian Networks}

The use of Bayesian networks is an approach to determine evidence for real world problems (Koller and Friedman, 2009). One obtains the desired likelihood within a directed acyclic graph, that consists of nodes, representing given observations and the conditional dependence between them, defined in a conditional probability table (CPT) or by the use of a conditional probability distribution.

An implementation towards modeling a general validation for the prediction of potential target objects is shown in (Zeisler et al., 2015). One of the key features of this approach is modeling a lane and the respective boundaries out of the ego motion parameters (velocity and yaw rate). Subsequently, the surrounding objects are related to these boundaries. Fig. 8 represents the derived and reduced network that would apply to determine the evidence for the example shown in Fig. 2, where the vehicle right to the ego lane attempts to change to the left. All nodes $X$ in this representation return a likelihood $P\left(X=x_{i}\right) \in[0,1]$ for their respective states $x_{i}$. The last node Object Relevance determines the likelihood $P(O R \mid L C, P o s)$ for the observed object as a relevant target vehicle. Position Classes (Pos) and Lane Change (LC) are the input nodes, providing evidence on the current position of the object (Pos $\in$ left, right, infront) and the likelihood for a lane change event ( $L C \in$ Left, Right, Follow).

In this example, the evidence in position is $P($ Pos $=$ right $)=1$ and the probability of a lane change is determined for a follow and left change event solely. The input for this event is determined from the node Lane Boundary Crossing Left ( $L B C_{l e f t}$ ), which evaluates the possible crossing of the boundary of our ego vehicle driving path. Table 5 provides the specification to derive the probability for a lane boundary crossing by

$$
\begin{aligned}
P(L B C)= & P(L E) \cdot P(T R)+ \\
& P(L E) \cdot P(\overline{T R})+ \\
& P(\overline{L E}) \cdot P(T R),
\end{aligned}
$$

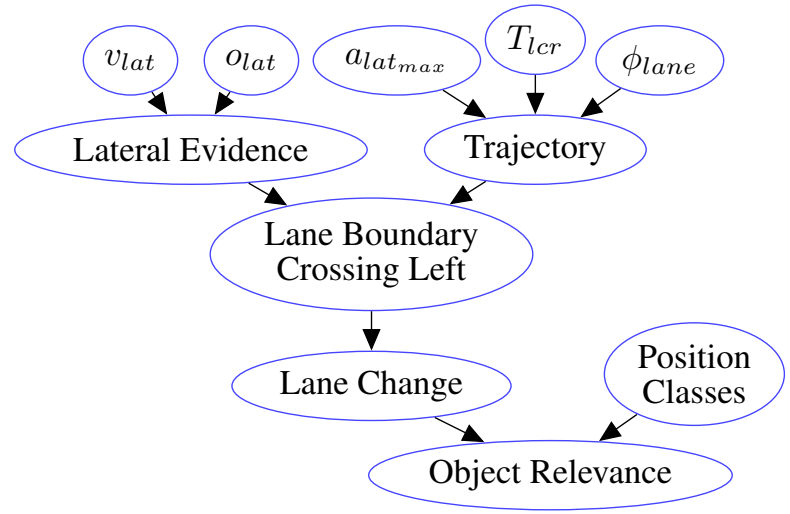

Figure 8: Representation of the reduced BN to determine the relevance of an object, located right to the ego vehicle. 
where Lateral Evidence ( $L E)$ and Trajectory $(T R)$ are input nodes with a single binary state, that could be true or false. The notation $P(X)$ relates to the true state $P(X)=$ True, whereas $P(\bar{X})$ relates to the false state $P(X)=$ False. Furthermore, the probabilities of the binary state sum to 1 per definition: $P(X)+$ $P(\bar{X})=1$. $L E$ and $T R$ are the behavior nodes for the implemented BN implementing the following geometric features $\left(G_{i}\right)$ as input to derive their probability (Kasper et al., 2012):

- Lateral offset $\left(o_{l a t}\right)$ towards the boundary

$$
o_{\text {lat }}=d-0.5 \cdot w,
$$

where $d$ is the distance of the object's rear center point to the derived boundary of the determined ego lane and $w$ is the width of the object.

- Lateral velocity $\left(v_{\text {lat }}\right)$ towards the boundary

$$
\vec{v}_{l a t}=R(\alpha) \cdot \vec{v},
$$

where $R(\alpha)$ denotes a two-dimensional rotation by the derived angle $\alpha$, resulting from the determined ego lane at the position of the object. The vector $\vec{v}$ represents the measured velocity of the object as seen from the ego vehicle. Finally, $v_{\text {lat }}$ is the lateral part of the derived vector.

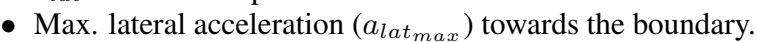

- Time to line crossing $\left(T_{l c r}\right)$ until the boundary.

- Relative angle $\left(\phi_{\text {lane }}\right)$ towards the boundary.

As the geometric features are of different units and ranges, logistic functions (sigmoid curves, Fig. 10a) are used to translate the features within given borders into probabilities. The resulting probability of the behavior nodes ( $B \in L E, T R$ ) determines, implementing the logistic function and assuming the independence of the features, to

$$
P\left(B \mid G_{i}\right)=\prod_{i=1}^{n} \frac{a_{i}}{a_{i}+\exp \left(b_{i} \cdot G_{i}\right)},
$$

where the geometric features $G_{i}$ are discretized and $a_{i}, b_{i}$ are the weighting parameters $(n=2 \forall L E, n=3 \forall T R$ ). They have been learned applying logistic regression and with the help of a test and training set, that has been labeled by an expert. A significant increase in performance detection, compared to manually chosen parameters has been shown (Schönert, 2015).

All geometric features $\left(a_{\text {lat }}\right.$ max $\left., T_{l c r}, \phi_{\text {lane }}\right)$ of Trajectory are derived, using an iterative lane change model and the input of $o_{\text {lat }}$ and the traveled distance. Therefore, the main performance of the detection depends on the inputs of Lateral Evidence. With regard to Eq. 8, the maximum probability $P(L B C)=1 \mathrm{might}$ be reached evenly from the single input of $L E$, if $T R$ is neglected. We will concentrate on the evaluation of $o_{\text {lat }}$ and $v_{\text {lat }}$ for further evaluation. Table 6 hands an impression for the resulting output from the example in Fig. 2, where the determined ego lane is assumed to be equal to the road lane (width: $3.5 \mathrm{~m}$ ) and the vehicles $(w=2.0 \mathrm{~m})$ are in the middle of their respective lanes at the start of the maneuver.

With given assumption $P(T R)=0$, the resulting probability $P(L E)$ is consequently passed through the Bayesian network (Fig. 8), resulting in the probability $P(O R)=P(L E)$ for the relevance of the object. Finally, we apply a threshold $S=65 \%$, that achieves a good balance between type I and type II errors, for the acceptance of the PTO as target object. Assuming best conditions, the resulting reaction time decreases to $t_{2} \approx 1.0 \mathrm{~s}$ for this example.

\begin{tabular}{cccc}
\hline Phase & olat $_{\text {l }}[\mathrm{m}]$ & $v_{\text {lat }}[\mathrm{m} / \mathrm{s}]$ & $P\left(L E \mid o_{\text {lat }}, v_{\text {lat }}\right)$ \\
\hline 1 (Fig. 2b) & 0.7 & 0.0 & 0.01 \\
2 (Fig. 2c) & 0.5 & -0.8 & 0.13 \\
3 (Fig. 2d) & -0.3 & -1.0 & 0.85 \\
\hline
\end{tabular}

Table 6: Example for the resulting probability of $L E$ during a lane change maneuver.

\subsection{Performance under Noise}

Generally, the geometric features $\left(G_{i}\right)$ are derived by the input of a sensor system ( $O$; consisting of a single or multiple sensors), providing observations of the surrounding objects and the sensors $(E)$, providing information of the ego vehicle, used to derive the ego lane of

$$
G_{i}=f(O, E) .
$$

The sensor data output is erroneous due to the sensor noise on measure and the noise effects in the data processing chain. We implement the approach characterized in (Weidl et al., 2005) to deal with noisy observations. To show the influence on the performance for the sensor under test, we proceed under the assumption, that the data of all sensors of the ego vehicle is free of bias: $\sigma_{O}=0$. The expectational value $(G)$ for a given feature determines with evidence of variance $\left(G_{\sigma^{2}}\right)$ and the measured value $\left(G_{\text {meas }}\right)$, assuming a Gaussian distribution and a zero mean error of all measurements to

$$
p\left(G_{\text {meas }} \mid G, G_{\sigma^{2}}\right)=N\left(G, G_{\sigma^{2}}\right) .
$$

With the help of intercausal inference, the BN approach (Fig. 9a) allows to determine the probabilities $P\left(G_{i, k}\right)$ for the distributed expectational value for every feature $G_{i}$, where $k$ depends on the amount of discretization steps $K$. Fig $9 \mathrm{~b}$ shows an example, where $K=7$ and the standard deviation is set to $\sigma=0.6$.

Determining the probability of a behavior node (Eq. 11) enhances to

$$
P\left(B \mid G_{i}\right)=\prod_{i=1}^{n} \sum_{k=1}^{K} P\left(G_{i, k}\right) \cdot \frac{a_{i}}{a_{i}+\exp \left(b_{i} \cdot G_{i, k}\right)} .
$$

Fig. 10a shows the resulting probability plot of $o_{\text {lat }}$ and $v_{\text {lat }}$ with assumed standard deviation. The derived probability $P(L E)$ is given in Fig. 10b. Blue areas reveal a low probability for the relevance of a PTO, whereas the red area shows a high potential, that the observed vehicle is a target object. Regarding the defined threshold $S$, one can find the curves for the detection of a target object from Fig. 10c, that is simulated under the assumption of
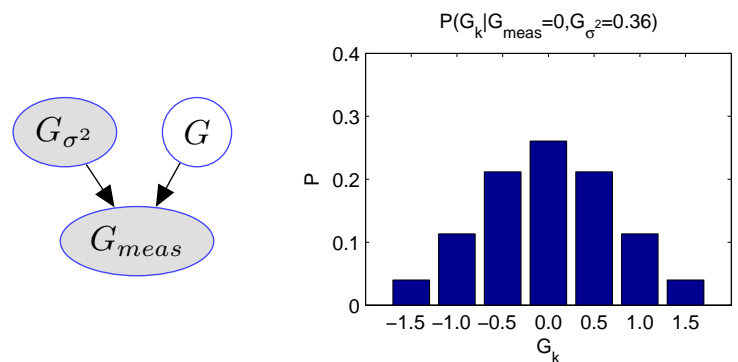

(a) Generic BN to handle (b) Propability for the expectational valmeasurement noise. Gray ues $G$ with given measurement $G_{m e a s}$ and nodes show evidence input. variance $G_{\sigma^{2}}$.

Figure 9: Handling noisy features. 


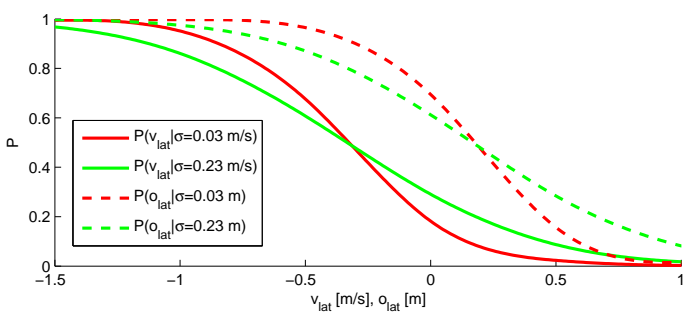

(a) Curves for the expectational values of $o_{\text {lat }}$ and $v_{\text {lat }}$ under noise.

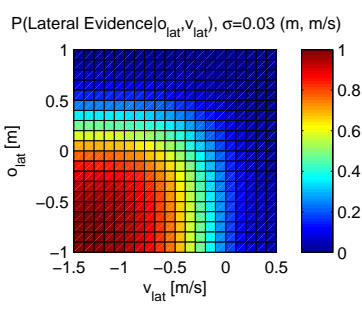

(b) Resulting $P(L E)$.

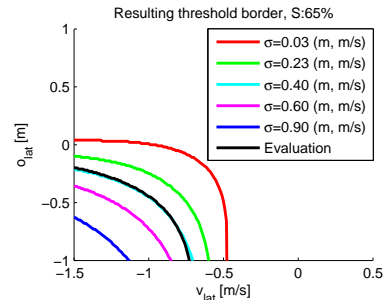

(c) Performance curves with given threshold $S$.
Figure 10: Probability under the presence of noise and resulting performance with given threshold.

a standard deviation distribution from $\sigma=0.03 \ldots 0.90$ for the geometric features. The red curve relates to the probability plot in Fig. 10b and gives an impression towards the values of the geometric features, required for a target object detection. If the lateral offset $o_{\text {lat }}$ equals $0.0 \mathrm{~m}$, the lateral velocity $v_{\text {lat }}$ must at least be $-0.8 m$ or less, otherwise $P(L E)<S$. With increasing sensor noise and higher standard deviation, the conditions, required to detect the target objects, increase as well. Furthermore, the reaction time increases. Assuming $\sigma=0.03$, it is approximately $t_{2} \approx 1.25 \mathrm{~s}$

With the use of the resulting uncertainties of chapter 3.2, one finds the resulting threshold curve in Fig. 10c, with the identifier Evaluation. As shown, it is similar to the curve, that results from an overall noise of $0.4 \mathrm{~m}$ for $o_{\text {lat }}$ and $v_{\text {lat }}$. The performance, under this assumptions, depends much more on the lateral velocity, observed for a vehicle during the lane change. Given best conditions $\left(v_{\text {lat }} \leq-1.3 \mathrm{~m} / \mathrm{s}\right)$, the reaction time will be $t_{2} \approx 1.5 \mathrm{~s}$, that equals a decrease of $40 \%$ compared to current state-of-theart systems. A lane change, characterized by slow lateral velocity will result in a much worse time, $t_{2} \approx 2.5 \mathrm{~s}$.

\section{CONCLUSION AND OUTLOOK}

In this paper we have shown the need for precise sensing technologies towards the use for future ADAS. Detecting cut-in maneuvers of traffic participants is one of the key goals to achieve autonomous driving functions. The laser scanner ScaLa has been presented and its sensing characteristics have been described. A field evaluation shows the sensor performance for position and velocity determination in object tracking. Compared to the algorithm, the determined performance shows potential, in particular for the derived velocities of the tracked objects.

Future work concentrates on the evaluation of a sensor-fusion setup and advanced approaches towards the target vehicle detection using Bayesian networks.

\section{ACKNOWLEDGEMENTS}

The authors would like to thank the colleagues at the Sensor departement at BMW and the System Development staff at Ibeo
Automotive Systems for their continuous support.

\section{REFERENCES}

Aeberhard, M., Rauch, S., Bahram, M., Tanzmeister, G., Thomas, J., Pilat, Y., Homm, F., Huber, W. and Kaempchen, N., 2015. Experience, results and lessons learned from automated driving on germany's highways. Intelligent Transportation Systems Magazine, IEEE 7(1), pp. 42-57.

Darms, M., Rybski, P., Baker, C. and Urmson, C., 2009. Obstacle detection and tracking for the urban challenge. Intelligent Transportation Systems, IEEE Transactions on 10(3), pp. 475-485.

Ibeo Automotive, 2015. ibeo.ADAS laser scanner: http://www.ibeo-as.com/ibeoadas.html, last visited: 11.04.2015.

ISO, 2009. Intelligent transport systems - full speed range adaptive cruise control (FSRA) systems - performance requirements and test procedures. ISO 22179:2009, International Organization for Standardization, Geneva, Switzerland.

Jochem, T., Pomerleau, D., Kumar, B. and Armstrong, J., 1995. Pans: a portable navigation platform. In: Intelligent Vehicles '95 Symposium., Proceedings of the, pp. 107-112.

Kasper, D., Weidl, G., Dang, T., Breuel, G., Tamke, A., Wedel, A. and Rosenstiel, W., 2012. Object-oriented bayesian networks for detection of lane change maneuvers. Intelligent Transportation Systems Magazine, IEEE 4(3), pp. 19-31.

Koller, D. and Friedman, N., 2009. Probabilistic graphical models: principles and techniques. MIT press.

Li, P., Alvarez, L. and Horowitz, R., 1997. Ahs safe control laws for platoon leaders. Control Systems Technology, IEEE Transactions on 5(6), pp. 614-628.

OXTS Ltd., 2015. OXTS RT 3000 v2 product overview: http://www.oxts.com/products/rt3000-family/, last visited: 14.04.2015.

Salvucci, D. D. and Liu, A., 2002. The time course of a lane change: Driver control and eye-movement behavior. Transportation Research Part F: Traffic Psychology and Behaviour 5(2), pp. $123-132$.

Schönert, F., 2015. Maschinelles Lernen zur Parametrierung eines probabilistischen Netzes für eine Erkennung von Fahrmanövern. Bachelorthesis, Technische Hochschule Deggendorf.

Velupillai, S. and Guvenc, L., 2009. Laser scanners for driverassistance systems in intelligent vehicles [applications of control]. Control Systems, IEEE 29(2), pp. 17-19.

Watanabe, T., Kishimoto, N., Hayafune, K., Yamada, K. and Maede, N., 1995. Development of an intelligent cruise control system. In: Steps Forward. Intelligent Transport Systems World Congress.

Weidl, G., Madsen, A. and Israelsson, S., 2005. Object-oriented bayesian networks for condition monitoring, root cause analysis and decision support on operation of complex continuous processes: Methodology \& applications. Institute for Systems Theory in Engineering University of Stuttgart, Hugin Expert A/S, ABB Group Services.

Winner, H., Hakuli, S. and Wolf, G., 2012. Handbuch Fahrerassistenzsysteme: Grundlagen, Komponenten und Systeme für aktive Sicherheit und Komfort. Springer.

Zeisler, J., Cherepanov, J. and Haltakov, V., 2015. A driving path based target object prediction. To appear in: Intelligent Vehicles Symposium (IV), 2015 IEEE. 